\title{
Loop Ileostomy and Colostomy-A Comparison between Supporting Plastic Rods and Epicutaneous or Subcutaneous Silicon Drains
}

\author{
Mike Ralf Langenbach ${ }^{1}$, Stefan Sauerland ${ }^{2}$, Eiyad Issa ${ }^{1}$, Claudia Nitschke ${ }^{3}$, Hubert Zirngibl ${ }^{3}$ \\ ${ }^{1}$ Helios St. Elisabeth Klinik Oberhausen, Department of Surgery II, University of \\ Witten/Herdecke, Oberhausen, Germany \\ ${ }^{2}$ Institute for Research in Operative Medicine (IFOM), University Witten/Herdecke, Cologne, Germany \\ ${ }^{3}$ Helios Klinikum Wuppertal, Department of Surgery II, University of Witten/Herdecke, Wuppertal, Germany \\ E-mail: mike-ralf.langenbach@helios-kliniken.de
}

Received March 21, 2011; revised May 23, 2011; accepted June 1, 2011

\begin{abstract}
Purpose: Beside the conventional plastic rods, different techniques and materials have been proposed in the last years to prevent the loop from retraction into the abdominal cavity. The aim of this retrospective comparative study was to assess three different techniques of loop support. Methods: The study included 65 patients who had loop ileostomy or colostomy formed. Depending on the decision of the operating surgeon, one of three techniques was chosen to fixate the stoma loop: an epicutaneous plastic rod (group 1, $\mathrm{n}=14$ ), an epicutaneous suture-fixated silicone drain (group 2, $\mathrm{n}=27$ ), or a subcutaneous silicone drain (group 3, $\mathrm{n}=$ 24). Results: The majority of patients (85\%) received loop ileostomy. Pain intensity was significantly $(\mathrm{p}=$ $0.0014)$ different among the three groups. A total of 19 patients (30\%) suffered a complication. There was a tendency towards less complications if the stoma was secured by a silicone drain with epicutaneous fixation. Comfort with stoma care was significantly different, with group 3 experiencing the best results. Conclusions: Using a subcutaneously tunnelled silicon drain as a stoma bridge results in less complications, less pain and higher satisfaction as compared to the conventional plastic rod. Conventional plastic rods should be avoided.
\end{abstract}

Keywords: Ileostomy, Colostomy, Drains, Plastic Rods

\section{Introduction}

Loop ileostomy and colostomy are frequently indicated in patients with acute or complicated intestinal diseases, such as acute neoplastic obstruction, inflammatory stenosis or perforation. Irregardless of the differences between ileostomy and colostomy [1-3], stomas usually cause a number of medical but also psychosocial problems. In two of the largest studies on this problem [4,5], local complications of minor severity were noted in one third of patients. According to Cottam, et al. [4], the most frequent complication was retraction of the stoma, which occurred in one quarter of patients. However, this figure needs to be interpreted with caution, because the definition of retraction is not straightforward.

Since many years plastic rods have been used to support a loop stoma and to prevent the loop from falling back into the abdominal cavity [6-8]. However, the use of rigid plastic rods is not without complications, mainly because the rod lies on the skin surface for one or two weeks after surgery, which can lead to skin maceration and local infection. Dissatisfaction with plastic rods is highly prevalent as evidenced by the large number of alternative techniques that were proposed in more recent years [9-16]. In most of these articles, the bridge which supported the stoma limbs was reinforced with a softer material, such as a Jackson-Pratt drain, a suction drain tube, or a Penrose drain. Some surgeons have even questioned whether the use of supporting bridges is necessary at all $[17,18]$.

Based on clinical experience, the technique of secureing loop ileostomies and colostomies at the authors' institution has been variable during the last years. The aim of this retrospective study therefore was to assess which technique of loop support provided the lowest rate of complications and the highest level of satisfaction for 
stoma therapist and patient.

\section{Material and Methods}

Consecutive patients with a first stoma between June 2008 and December 2009 were studied. Stoma construction followed either an emergency or an elective surgical procedure. Depending on the decision of the operating surgeon, one of three stoma techniques was chosen: a conventional epicutaneous plastic rod (group 1), an epicutaneous suture-fixated silicone drain (group 2), or a silicone drain placed in a subcutaneous tunnel (group 3). The selection of surgical technique depended more on the surgeon's personal expertise than on the patient's characteristics.

The steps in performing a loop colo- or ileostomy with a suprafascial bridge device were similar to those generally described in international textbooks. After a suitable incision in the abdominal wall had been made, the colic or ileic loop was gently pulled through the opening and a small incision was made in the mesentery. In group 1, a conventional plastic rod (ConvaTec ${ }^{\circledR}$, Coloplast Inc., Hamburg, Germany) measuring 7 or $10 \mathrm{~cm}$ in length was placed through the mesenteric window. At both ends of the rod small wings were opened, so that the rod could not slip through the mesentery.

In groups 2 and 3, a 21 Charrière silicone drain was used. Both ends were pointed to ease tissue penetration. In group 2, after placement through the mesentery, the ends of the silicon drain were fixed with nonresorbable polyamid sutures (Ethilone, 3.0; Ethicon, Inc.) to the skin at the incisional site. The stitches were inside the range of the stoma appliance. When the surgeon decided to secure the stoma with a subcutaneous silicone drain (group 3), the silicone drain was cut to a length of about $20 \mathrm{~cm}$. After being placed through the mesentery, both ends of the drain were subcutaneously tunnelled, were brought out and fixed with a stitch. Entry and exit point of the drain were just beyond the circumference of the stoma flange. Finally, both end of the drain were shortened to the level of the skin.

Whilst in the hospital the patients were regularly reviewed and stomata were assessed by a surgeon and stoma therapist. Using standardized forms, we recorded demographic characteristics, surgical indication, and type of surgery. At the first, third, fifth and tenth postoperative day, the severity of local pain (using a 0 to 10 visual analogue scale [VAS]), the occurrence of local complications, and the level of satisfaction of stoma therapist and patient were recorded. The definition of complications was adapted from other studies and required the presence of patient-reported symptoms, or the necessity of specific therapy (local or systemic). Follow up was continued until stoma support was removed, which took place between the seventh and tenth postoperative day. All data collected in the patients' charts were retrospecttively reviewed and analysed.

\section{Statistical Analysis}

Differences in baseline and outcome parameters between the three groups were statistically assessed using analysis of variance (ANOVA) or chi square testing. In order to account for the fact that pain scores represented repeated measures of the same individual, these were analyzed with a general linear model followed by post hoc Tukey tests. Significance was defined as a p-value smaller than 0.05 .

\section{Results}

Among the 65 patients, there were 55 (85\%) loop ileostomies and 10 (15\%) loop colostomies. In colostomy patients, a plastic rod was used more frequently. With regard to other baseline variables, the groups were more or less comparable (Table 1).

As shown in Figure 1, pain was clearly different among the three groups $(p=0.014)$. Group-wise comparisons indicated that pain was significantly lower in the group with subcutaneous silicone drains than in each of the other two groups: $\mathrm{p}=0.008$ vs. plastic rod and $\mathrm{p}<$ 0.001 vs. silicone drain on skin. No patient required analgesics because of local pain.

A total of 19 patients (29\%) suffered a complication. There were slightly less complications in patients who had their stoma secured by a superficially placed silicone drain (Table 2), but this difference was not significant. However, the severest complications (arrosion of the intestine) were all seen exclusively in the group with conventional plastic rods. All wound infections in the group with subcutaneously tunnelled silicon drains were superficial and mild.

From the viewpoint of the stoma therapist, comfort and ease of stoma care was significantly different among the groups, as the group with subcutaneously placed silicon drains showed the best results. Since entry and exit site of the silicon drain in this group were outside the diameter of the stomal flange, changing the stoma bag did not interfere with any type of rod or drain.

\section{Discussion}

The creation of a loop ileo- or colostomy strongly influences the patients' quality of life after an operation. Furthermore, patient cooperation is necessary for correct stoma care. Therefore, it is essential that no complication decreases the patients' trust in the normal functioning of 
Table 1. Demographic baseline characteristics.

\begin{tabular}{|c|c|c|c|c|}
\hline & Conventional plastic rod & Silicon drain on skin surface & $\begin{array}{c}\text { Silicon drain with } \\
\text { subcutaneous tunnel }\end{array}$ & P-value \\
\hline Number of patients & 14 & 27 & 24 & \\
\hline Age (in years) with range & $63(16) 31-81$ & 66 (12) $27-86$ & 73 (11) $40-87$ & $0.028^{\mathrm{a})}$ \\
\hline Gender (proportion males) & $9(64 \%)$ & $15(56 \%)$ & $15(63 \%)$ & $0.82^{\mathrm{b})}$ \\
\hline Underlying disease & & & & $0.15^{\mathrm{b})}$ \\
\hline Rectal cancer & $6(43 \%)$ & $16(59 \%)$ & $16(67 \%)$ & \\
\hline Colon cancer & $1(7 \%)$ & $3(11 \%)$ & $4(17 \%)$ & \\
\hline Intestinal ischemia & $2(14 \%)$ & $3(11 \%)$ & $1(4 \%)$ & \\
\hline Inflammatory bowel disease & 0 & $2(7 \%)$ & $1(4 \%)$ & \\
\hline Sacral pressure ulcer & 0 & $1(4 \%)$ & $2(8 \%)$ & \\
\hline Diverticulitis & $1(7 \%)$ & $1(4 \%)$ & 0 & \\
\hline Other $^{c}$ ) & $4(27 \%)$ & $1(4 \%)$ & 0 & \\
\hline Type of stoma & & & & $0.05^{\mathrm{b})}$ \\
\hline Loop ileostomy & $11(79 \%)$ & $24(89 \%)$ & $20(83 \%)$ & \\
\hline Loop colostomy & $3(21 \%)$ & $3(11 \%)$ & $4(17 \%)$ & \\
\hline
\end{tabular}

Data are means \pm standard deviations (with ranges) or counts (with percentages); ${ }^{\text {a) }}$ by ANOVA, ${ }^{\text {b) }}$ by Chi square test, ${ }^{\text {c) }}$ This category included rectal impalement injury, advanced prostatic cancer, rectocele, complicated ileus, and multiple sclerosis.

Table 2. Clinical results.

\begin{tabular}{|c|c|c|c|c|}
\hline & Conventional plastic rod & $\begin{array}{l}\text { Silicon drain on } \\
\text { skin surface }\end{array}$ & $\begin{array}{l}\text { Silicon drain with } \\
\text { subcutaneous tunnel }\end{array}$ & P-value \\
\hline Number of patients & 14 & 27 & 24 & \\
\hline Any complication & $7(50 \%)$ & $8(30 \%)$ & $4(17 \%)$ & 0.41 \\
\hline Local complications & & & & NA \\
\hline Arrosion of intestine & $5^{c)}$ & & & \\
\hline Partial necrosis of intestine & 1 & 2 & & \\
\hline Intestinal malperfusion & 1 & 1 & & \\
\hline Fixation suture torn out & & 1 & & \\
\hline Infection at drain entry site & & 1 & 2 & \\
\hline Small stoma prolapse & & & 1 & \\
\hline Small parastomal hernia & & & 1 & \\
\hline Local swelling & & 3 & & \\
\hline Comfort of stoma care & & & & 0.001 \\
\hline Good & $2(14 \%)$ & $12(44 \%)$ & $24(100 \%)$ & \\
\hline Average & $5(36 \%)$ & $9(33 \%)$ & 0 & \\
\hline Bad & $7(50 \%)$ & $6(22 \%)$ & 0 & \\
\hline
\end{tabular}

Data are means \pm standard deviations (with ranges); ${ }^{\text {a) }}$ by ANOVA, ${ }^{\text {b) }}$ by Chi square test, ${ }^{\text {c) }}$ On day 1,3 (three cases) and 5 after stoma creation.

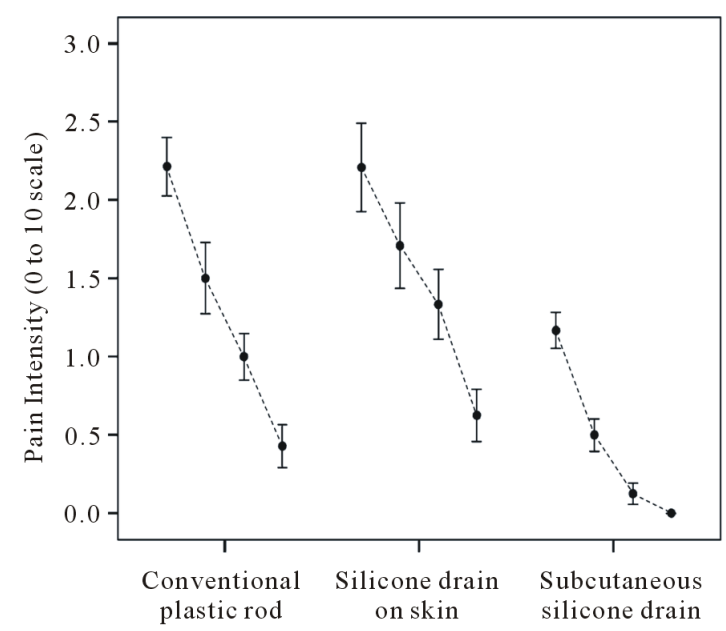

Figure 1. Local pain on day 1, 3, 5, and 7 after different types of stoma creation Data are means with error bars indicating one standard error. the stoma. In this study, complications were more frequent and more severe in patients with a conventionally secured stoma. In order to avoid arrosion of the intestinal loop, the use of softer material for the stoma bridge appears necessary. Nevertheless, complications were still quite common in the two groups, where a silicon drain was used instead of a plastic rod. This shows the need for further improvements in surgical techniques.

The complication rate of this study (29\%) is well in line with previous research [4,19-26]. In a large registry study, Cottam, et al. identified $34 \%$ of 3970 stomas as problematic [4]. In that study, type of stoma and gender of the patient were described as significant risk factors, but also the height of the stoma was predictive for complications. In our experience, securing the stoma loop with a silicon drain effectively prevented any retraction of the loop into the wound. Although stoma height is 
difficult to measure quantitatively, the subcutaneous position of a silicon drain is fully sufficient to support the loop, which then guarantees for correct emptying of intestinal contents into the stoma appliance.

Using a subcutaneously tunnelled suction drain as a stoma bridge was first described in 2008 by Harish [13]. As the two main advantages of this technique, he considered the quick availability of suction drains in operating theatre and the ease of stoma care device application. In the present study, surgical techniques were exactly the same as described by Harish, except for the fact that he used tubes of 16 or 18 French, whereas a calibre of 21 French was preferred by us. This difference can probably be best explained by the larger body size of Europeans as compared to Indians, in whom Harish developed the technique.

Already in 1984, Jenkinson, et al. proposed to use the subcutaneous layer for placement of the stoma bridge [27]. In a more recent study, Branco, et al. [12] implanted a conventional plastic bridge device in the subcutaneous tissue. In this technique, there is no need for additional skin incisions, which clearly reduces the risk of wound infection. Among the 19 cases reported by Branco, et al., there was not a single infection, but apparently pain was a problem, as two patients requested analgesics. Of note, pain during bridge removal was not reported in the study by Branco, et al., because the plastic rod remained in place until stoma reversal. However, a permanent plastic rod in the abdominal wall may cause discomfort to the patient. Since silicone is a softer material than plastic, pain was clearly reduced in the present study. It is quite interesting that a silicon drain causes less pain when subcutaneously tunnelled than being placed on the skin. Apparently, the stitches for securing the drain must also be considered a potential source of pain, because the intestinal loop may pull on these stitches.

An important strength of the present study is that it represents a comparative design including three comparison groups and assessment of patient-reported outcomes. Although the assignment of patients into the three groups was not randomised, the potential for selection bias was very small, because during the study period most surgeons performed only one of the three techniques. Usually, evidence derived from prospectively controlled trials allows for more valid conclusions than do case series without a control group. To some extent the lack of comparative studies is certainly responsible for the large variety in surgical techniques in this field and the lack of a consensus on the best technique [28].

\section{Conclusions}

In summary, our data implicate that a subcutaneously tunnelled silicon drain provides the lowest rate of complications and pain and the highest level of satisfaction for patient and stoma therapist. Plastic rods should be avoided.

\section{References}

[1] A. W. Gooszen, R. H. Geelkerken, J. Hermans, M. B. Lagaay and H. G. Gooszen, "Quality of Life with a Temporary Stoma: Ileostomy vs. Colostomy,” Diseases of the Colon \& Rectum, Vol. 43, No. 5, 2000, pp. 650-655. doi:10.1007/BF02235581

[2] N. S. Williams, D. G. Nasmyth, D. Jones and A. H. Smith, "Defunctioning Stomas: A Prospective Controlled Trial Comparing Loop Ileostomy with Loop Transverse Colostomy,” British Journal of Surgery, Vol. 73, No. 7, 1986, pp. 566-570. doi:10.1002/bjs.1800730717

[3] S. D. Wexner, D. A. Taranow, O. B. Johansen, F. Itzkowitz, N. Daniel, J. J. Nogueras and D. G. Jagelman, "Loop Ileostomy is a Safe Option for Fecal Diversion," Diseases of the Colon \& Rectum, Vol. 36, No. 4, 1993, pp. 349-354. doi:10.1007/BF02053937

[4] J. Cottam, K. Richards, A. Hasted and A. Blackman, "Results of a Nationwide Prospective Audit of Stoma Complications within 3 Weeks of Surgery," Colorectal Disease, Vol. 9, No. 9, 2007, pp. 834-838. doi:10.1111/j.1463-1318.2007.01213.x

[5] J. J. Park, A. Del Pino, C. P. Orsay, R. L. Nelson, R. K. Pearl, J. R. Cintron and H. Abcarian, "Stoma Complications: The Cook County Hospital Experience,” Diseases of the Colon \& Rectum, Vol. 42, No. 12, 1999, pp. 1575 -1580. doi:10.1007/BF02236210

[6] S. M. Poticha, "A New Technic for Loop Colostomy with Use of a Plastic Bridge,” American Journal of Surgery, Vol. 127, No. 5, 1974, pp. 620-621. doi:10.1016/0002-9610(74)90333-X

[7] J. M. Corman and D. B. Odenheimer, "Securing the Loop-Historic Review of the Methods Used for Creating a Loop Colostomy," Diseases of the Colon \& Rectum, Vol. 34, No. 11, 1991, pp. 1014-1021. doi:10.1007/BF02049967

[8] P. M. Go, N. H. Vaessen, C. J. van Duin and J. Lens, “A Plastic Rod to Facilitate Longitudinal Incision of the Bowel. An Inexpensive and Practical Device,” Diseases of the Colon \& Rectum, Vol. 29, No. 10, 1986, p. 674. doi:10.1007/BF02560338

[9] R. A. Cochrane, D. J. Hay and A. F. Jones, “A Better Bridge for Loop Stomas,” British Journal of Surgery, Vol. 83, No. 3, 1996, p. 365. doi:10.1002/bjs.1800830322

[10] R. J. Fitzgibbons, Jr., G. D. Schmitz and R. T. Bailey, Jr., "A Simple Technique for Constructing a Loop Enterostomy which Allows Immediate Placement of an Ostomy Appliance," Surgery, gynecology \& obstetrics, Vol. 164, No. 1, 1987, pp. 78-80.

[11] S. W. Atkinson and P. G. Bentley, "Subcutaneous Bridge Support for Defunctioning Loop Colostomy,” British Journal of Surgery, Vol. 83, No. 10, 1996, p. 1458. doi:10.1002/bjs. 1800831042 
[12] A. M. Branco and A. C. Saraiva, "Loop Colostomy with a Suprafascial Bridge Device,” Digestive Surgery, Vol. 26, 2009, No. 4, pp. 282-284.

[13] K. Harish, "The Loop Stoma Bridge-A New Technique," Journal of Gastrointestinal Surgery, Vol. 12, No. 5, 2008, pp. 958-961. doi:10.1007/s11605-007-0413-7

[14] J. W. Nunoo-Mensah, A. Chatterjee, D. Khanwalkar and D. G. Nasmyth, "Loop Ileostomy: Modification of Technique,” Surgeon, Vol. 2, No. 5, 2004, pp. 287-291. doi:10.1016/S1479-666X(04)80099-4

[15] E. T. Goldstein and P. R. Williamson, "A More Functional Loop Ileostomy Rod," Diseases of the Colon \& Rectum, Vol. 36, No. 3, 1993, pp. 297-298. doi:10.1007/BF02053516

[16] G. Atkin, M. A. Scott, P. Mathur and I. C. Mitchell, "The Rectus Sling to Prevent Loop Colostomy Retraction: A Case Series," International Seminars In Surgical Oncology, Vol. 2, 2005, p. 22. doi:10.1186/1477-7800-2-22

[17] M. Speirs, E. Leung, D. Hughes, I. Robertson, L. Donnelly, I. Mackenzie and A. Macdonald, "Ileostomy Rod -Is it a Bridge too Far?” Colorectal Disease, Vol. 8, No. 6, 2006, pp. 484-487. doi:10.1111/j.1463-1318.2005.00923.X

[18] J. A. Unti, H. Abcarian, R. K. Pearl, C. P. Orsay, R. L. Nelson, M. L. Prasad, B. Duarte, M. M. Leff and A. B. Tan, "Rodless Endloop Stomas. Seven-Year Experience," Diseases of the Colon \& Rectum, Vol. 34, No. 11, 1991, pp. 999-1004. doi:10.1007/BF02049964

[19] R. J. Aitken, P. J. Stevens, N. du Preez and M. S. Elliot, "Raising a Colostomy-Results of a Prospective Surgical Audit," International Journal of Colorectal Disease, Vol. 1, No. 4, 1986, pp. 244-247. doi:10.1007/BF01648346

[20] P. J. Arumugam, L. Bevan, L. Macdonald, A. J. Watkins, A. R. Morgan, J. Beynon and N. D. Carr, "A Prospective Audit of Stomas-Analysis of Risk Factors and Complications and their Management," Colorectal Disease, Vol. 5, No. 1, 2003, pp. 49-52. doi:10.1046/j.1463-1318.2003.00403.x
[21] D. A. Harris, D. Egbeare, S. Jones, H. Benjamin, A. Woodward and M. E. Foster, "Complications and Mortality Following Stoma Formation," Annals of The Royal College of Surgeons of England, Vol. 87, No. 6, 2005, pp. 427-431. doi:10.1308/003588405X60713

[22] M. Caricato, F. Ausania, V. Ripetti, F. Bartolozzi, G. Campoli and R. Coppola, "Retrospective Analysis of Long-Term Defunctioning Stoma Complications after Colorectal Surgery,” Colorectal Disease, Vol. 9, No. 6, 2007, pp. 559-561. doi:10.1111/j.1463-1318.2006.01187.x

[23] I. Robertson, E. Leung, D. Hughes, M. Spiers, L. Donnelly, I. Mackenzie and A. Macdonald, "Prospective Analysis of Stoma-Related Complications," Colorectal Disease, Vol. 7, No. 3, 2005, pp. 279-285. doi:10.1111/j.1463-1318.2005.00785.x

[24] M. Gutman, O. Kaplan, Y. Skornick, F. Greif, P. Kahn and R. R. Rozin, "Proximal Colostomy: Still an Effective Emergency Measure in Obstructing Carcinoma of the Large Bowel," Journal of Surgical Oncology, Vol. 41, No. 3, 1989, pp. 210-212.

[25] J. C. Duchesne, Y. Z. Wang, S. L. Weintraub, M. Boyle and J. P. Hunt, "Stoma Complications: A Multivariate Analysis,” American Journal of Surgery, Vol. 68, No. 11, 2002, pp. 961-966.

[26] R. K. Pearl, M. L. Prasad, C. P. Orsay, H. Abcarian, A. B. Tan and M. T. Melzl, "Early Local Complications from Intestinal Stomas,” Archives of Surgery, Vol. 120, No. 10, 1985, pp. 1145-1147.

[27] L. R. Jenkinson, P. W. J. Houghton, K. V. Steele, L. A. Donaldson and M. K. H. Crumplin, "The Biethium Bridge-an Advance in Stoma Care," Annals of The Royal College of Surgeons of England, Vol. 66, 1984, pp. 420-422.

[28] O. Kaidar-Person, B. Person and S. D. Wexner, “Complications of construction and closure of temporary loop ileostomy," Journal of the American College of Surgeons, Vol. 201, No. 10, 2005, pp. 759-773. doi:10.1016/j.jamcollsurg.2005.06.002 